\title{
(6) OPEN ACCESS \\ Changes in multiple cytokine concentrations in the aqueous humour of neovascular age-related macular degeneration after 2 months of ranibizumab therapy
}

\author{
Shinichi Sakamoto, ${ }^{1}$ Hidenori Takahashi, ${ }^{1,2,3}$ Xue Tan, ${ }^{2}$ Yuji Inoue, ${ }^{1,2}$ Yoko Nomura, ${ }^{2}$ \\ Yusuke Arai, ${ }^{1}$ Yujiro Fujino, ${ }^{3}$ Hidetoshi Kawashima, ${ }^{1}$ Yasuo Yanagi, ${ }^{4,5,6}$
}

\begin{abstract}
- Additional material is published online only. To view please visit the journal online (http://dx.doi.org/10.1136/ bjophthalmol-2017-310284).

${ }^{1}$ Department of Ophthalmology, Jichi Medical University,

Shimotsuke, Tochigi, Japan ${ }^{2}$ Department of Ophthalmology, Graduate School of Medicine, The University of Tokyo, Bunkyoku, Tokyo, Japan

${ }^{3}$ Department of Ophthalmology, Japan Community Health Care Organization Tokyo Shinjuku Medical Center, Tokyo, Japan ${ }^{4}$ Department of Medical Retina, Singapore National Eye Centre Singapore, Singapore ${ }^{5}$ Department of Medical Retina, Singapore Eye Research Institute, Singapore, Singapore ${ }^{6}$ Department of Ophthalmology and Visual Sciences Academic Clinical Program, Duke-NUS, Medical School, National University of Singapore, Singapore, Singapore
\end{abstract}

\section{Correspondence to} Professor Hidenori Takahashi, Department of Ophthalmology, Jichi Medical University, 33111 Yakushiji, Shimotsuke-shi, Tochigi 329-0431, Japan; takahah-tky@umin.ac.jp

Received 9 February 2017 Revised 6 July 2017

Accepted 11 July 2017 Published Online First 1 August 2017

\begin{abstract}
Purpose To determine changes in multiple cytokine concentrations in the anterior chamber during the induction phase of ranibizumab treatment in patients with neovascular age-related macular degeneration (AMD).
\end{abstract}

Methods This prospective study included 48 treatmentnaïve neovascular AMD eyes of 48 patients who received three consecutive monthly injections of ranibizumab at the Japan Community Health Care Organization Tokyo Shinjuku Medical Center between November 2010 and August 2012. We collected $\sim 0.2 \mathrm{~mL}$ aqueous humour before the first and third ( 2 months later) injections. Controls were 80 eyes with cataracts without retinal disease. The cytokines $\mathrm{C}-\mathrm{X}-\mathrm{C}$ motif chemokine ligand 1 (CXCL1), interferon- $\gamma$-induced protein 10 (IP-10), C-X-C motif chemokine ligand 12 (CXCL12), C-X-C motif chemokine ligand 13 (CXCL13), monocyte chemoattractant protein 1 (MCP-1), CCL11, C-C motif chemokine ligand 11 (CCL11), interleukin-6 (IL-6), interleukin-10 (IL-10) and matrix metalloproteinase 9 (MMP-9) were analysed using multiplex cytokine assays. Results Mean ages of the patients with AMD and controls were 73 and 75 years, respectively, and 31 (65\%) and 37 (46\%) subjects were men, respectively. Polypoidal choroidal vasculopathy was found in 27 eyes (56\%). Mean concentrations of cytokines in aqueous humour in patients with neovascular AMD before the first and third ranibizumab injections were as follows (in pg/mL): CXCL1, 8.4 and 3.3; IP-10, 110 and 55; CXCL12, 480 and 240; CXCL13, 9.2 and 2.6; MCP-1, 620 and 220; CCL11, 7.1 and 2.8; IL-6, 5.9 and 1.6; IL-10, 0.15 and 0.015 (all $p<0.0001$ ), and MMP-9, 0.92 and $1.5(p=0.0216)$, respectively. Concentrations of all cytokines decreased significantly after two consecutive ranibizumab injections, except for MMP-9, which increased significantly.

Conclusions After two monthly consecutive antivascular endothelial growth factor injections, inflammatory cytokine levels in the aqueous humour of the eyes with AMD were strongly suppressed, while MMP-9 levels increased.

\section{INTRODUCTION}

Angiogenesis and vascular hyperpermeability in neovascular age-related macular degeneration (AMD) are mainly caused by vascular endothelial growth factor (VEGF) and other inflammatory cytokines. ${ }^{1}$ Anti-VEGF therapy blocks VEGF to inhibit both vascular hyperpermeability and inflammation.
Previous investigations have reported that eyes with AMD have elevated concentrations of many cytokines, such as interferon- $\gamma$-induced protein 10 (IP-10), monocyte chemoattractant protein 1 (MCP-1), C reactive protein, intercellular adhesion molecule 1 and vascular cell adhesion molecule $1 .^{2}$ These cytokines also modify the activity of choroidal neovascularisation (CNV) in neovascular AMD. MCP-1, the best-studied cytokine in exudative $A M D$, recruits inflammatory monocytes to inflamed tissue. Subsequently, monocytes positively and negatively control inflammation by producing other angiogenic/inflammatory cytokines, such as VEGF and interleukin (IL)- $6,{ }^{4}$ and anti-inflammatory cytokines, such as IL-10. Other important inflammatory cytokines involved in AMD include $\mathrm{C}-\mathrm{X}-\mathrm{C}$ motif chemokines, such as $\mathrm{C}-\mathrm{X}-\mathrm{C}$ motif chemokine ligand 1 (CXCL1), CXCL10 (IP-10), CXCL12 and CXCL13, which also recruit leucocytes and promote angiogenesis. ${ }^{5}$ Some of these $\mathrm{C}-\mathrm{X}-\mathrm{C}$ cytokines are expressed in retinal pigment epithelium and are upregulated in AMD. Importantly, other than cytokines and chemokines, tissue proteases, such as matrix metalloproteinase 9 (MMP-9), are overexpressed in CNV and accelerate CNV growth. ${ }^{6}$ However, there is little information on the intraocular concentrations of these molecules that are considered to play pivotal roles in neovascular AMD.

Several studies have investigated changes in aqueous humour cytokine concentrations after the intravitreal administration of anti-VEGF drugs. In a previous study, a reduction was observed in the concentration of VEGF, with a concomitant increase in platelet-derived growth factor AA (PDGF-AA), using a flexible bevacizumab treatment regimen of one plus pro re nata (PRN). ${ }^{7}$ Another study investigated the change in aqueous humour cytokine concentrations 2 days after bevacizumab injection and demonstrated that the concentration of VEGF tended to decrease, whereas IL- 6 and IL- 8 levels increased. ${ }^{8}$ Although these pilot studies provided important information regarding changes in cytokine levels after the injection of an anti-VEGF drug, several important issues remain unclear. First, what are the changes in aqueous humour cytokine levels after monthly dosing for 3 months? There is evidence to suggest that a treatment regimen that employs monthly dosing for 3 months can achieve better treatment outcomes compared with the one plus PRN regimen ${ }^{9}$; thus, most physicians use three 
injections for the induction phase of anti-VEGF therapy. Second, is there an association between cytokine levels and anatomical outcomes? A previous study has suggested that VEGF and PDGF might be associated with disease activity $^{7}$; thus, are any other cytokines associated with anatomical outcomes? It has become generally accepted that there is a drastic change in the plasma concentrations of various inflammatory cytokines after systemic anti-VEGF drug administration. Several studies have indicated that some of these cytokines can be used as potential biomarkers to predict the treatment outcome of anti-VEGF therapy for solid tumours. ${ }^{10}$ Unfortunately, in the previous analysis, among the 29 cytokines examined, only VEGF, PDGF-AA, IL-6, IP-10 and MCP-1 were at measurable levels. ${ }^{7}$ Additionally, the methods used in these previous studies were inappropriate, ${ }^{11}$ and further studies are needed to confirm the findings. Moreover, previous studies have included only a limited number of patients (28-37 cases). To address these gaps in knowledge, we conducted the current analysis.

For this purpose, we compared the concentrations of cytokines before the first and third intravitreal ranibizumab injections. Based on the previous studies and our recent experiments, ${ }^{12}$ we chose to analyse the following cytokines: CXCL1, IP-10, CXCL12, CXCL13, MCP-1, C-C motif chemokine ligand 11 (CCL11), IL-6, IL-10 and MMP-9. Our investigation has the following advantages: (1) we investigated the largest number of patients thus far (48 treatment-naïve neovascular AMD eyes), and (2) we employed the standardised anti-VEGF treatment regimen of monthly dosing for 3 months.

\section{METHODS}

\section{Study design and approval}

This prospective study followed the tenets laid out in the Declaration of Helsinki. Informed consent was obtained from all patients. Institutional review board approval was obtained from the Japan Community Health Care Organization Tokyo Shinjuku Medical Center.

\section{Procedure}

The present study included 48 treatment-naïve neovascular AMD eyes of 48 patients. They first received three consecutive monthly injections of ranibizumab between November 2010 and August 2012 at Japan Community Health Care Organization Tokyo Shinjuku Medical Center. Controls were 80 cataract surgery eyes without fundus disease.

Approximately $0.2 \mathrm{~mL}$ of aqueous humour was collected just before the first and third ranibizumab injections. At the beginning of cataract surgery, a sample of undiluted aqueous humour (usually a volume of about $0.2 \mathrm{~mL}$ ) was manually aspirated into a disposable syringe, immediately transferred to a sterile tube and stored at $-80^{\circ} \mathrm{C}$ until required.

The concentrations of the following cytokines were determined using a multiplex cytokine assay (Filgen, Aichi, Japan) according to the manufacturer's instructions: CXCL1, IP-10, CXCL12, CXCL13, MCP-1, CCL11, IL-6, IL-10 and MMP-9. Their detection limits were $1.25,2.76,2.87,0.76,0.95,0.43$, $0.41,0.15$ and $0.57 \mathrm{pg} / \mathrm{mL}$, respectively. The measurements were performed twice for each sample and an average was calculated.

The concentration of VEGF was measured using an ELISA kit (R\&D Systems, Minneapolis, Minnesota, USA) according to the manufacturer's instructions, when the sample volume was enough to measure VEGF concentration. The VEGF kit permitted the detection of two of the four VEGF isoforms (VEGF121 and VEGF165). The detection limit was $2.2 \mathrm{pg} / \mathrm{mL}$.
Fluorescein angiography was performed routinely in neovascular AMD cases, except in patients with contraindications due to drug allergy, liver dysfunction or recent cerebrovascular events, to diagnose AMD and to discriminate type 1 and 2 CNV. Indocyanine green angiography, together with fluorescein angiography, was performed to identify polypoidal choroidal vasculopathy (PCV). Each patient underwent spectral domain optical coherence tomography (SD-OCT; Cirrus HD-OCT Model 4000 (Carl Zeiss Meditec AG, Jena, Germany)) at every visit. Central retinal thickness (CRT) was defined as the distance from the inner limiting membrane to Bruch's membrane. Each thickness was measured manually at the foveal centre using the OCT calliper function. Axial length was examined by A-mode ultrasonography (UD-6000, Tomey, Aichi, Japan). Posterior vitreous detachment (PVD) was examined by B-mode ultrasonography (UD-6000), as previously detailed elsewhere. ${ }^{13}{ }^{14}$ Briefly, the mobility of the posterior vitreous during ocular saccades was examined using the 'through the lid contact' technique. If the posterior vitreous was detached from the retinal surface and motile with eye movements, the eyes were categorised as having complete PVD (PVD group); otherwise, the eyes were categorised as the without PVD group. The without PVD group included eyes with partial PVD and no PVD.

\section{Statistical analysis}

Statistical analysis was performed using JMP Pro V.11.2.0 software (SAS Institute, Cary, North Carolina, USA). Categorical data were assessed using $\mathrm{X}^{2}$ tests, and continuous variables were assessed using Student's t-tests. Cytokine concentration changes were analysed by paired t-tests after logarithmic transformation. When concentrations were compared between AMD and control subjects, the associations between baseline characteristic factors, such as age, sex, axial length and the presence or absence of PVD, were corrected by multiple regression analysis. Statistical models using log-transformed concentrations were examined because of the skewed distribution of this variable. After discussion with a statistician, Bonferroni correction was not performed because this was a hypothesis-generating study whose purpose was to identify a possible association between AMD and cytokines. When concentrations were compared between whether eyes were still wet at the third injection or not, and between whether they experienced recurrence within 4 months of the first injection or not, the associations between baseline characteristics, such as age, sex, axial length and the presence or absence of PVD, were corrected by multiple regression analysis. To select the explanatory variables for cytokine concentrations,

\begin{tabular}{llll} 
Table 1 & Demographic characteristics of the patients & \\
\hline & AMD & Control & p Value \\
\hline $\mathrm{n}$ & 48 & 80 & \\
Male, $\mathrm{n}(\%)$ & $31(64.6)$ & $37(46.3)$ & $0.043^{*}$ \\
Age, years; mean \pm SD & $72.9 \pm 7.7$ & $74.8 \pm 6.6$ & $0.17 \dagger$ \\
Axial length, mm; mean \pm SD & $23.8 \pm 1.5$ & $23.2 \pm 1.1$ & $0.041 \dagger$ \\
PVD, $\mathrm{n}(\%)$ & $22(45.3)$ & $51(63.8)$ & $0.048^{*}$ \\
Disease type & & & \\
Typical AMD, $\mathrm{n}(\%)$ & $21(43.8)$ & & \\
PCV, $\mathrm{n}(\%)$ & $27(56.3)$ & & \\
RAP, $\mathrm{n}(\%)$ & $0(0)$ & & \\
\hline
\end{tabular}

${ }^{*} \mathrm{X}^{2}$ test.

tStudent's t-test.

AMD, age-related macular degeneration; PCV, polypoidal choroidal vasculopathy; PVD, posterior vitreous detachment; RAP, retinal angiomatous proliferation. 
A

B $\quad$ C

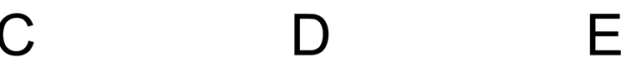

CXCL1

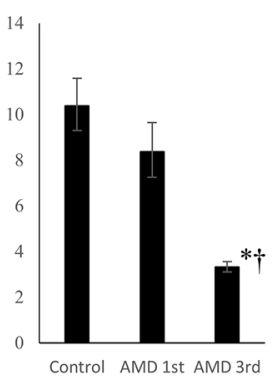

F

CCL11

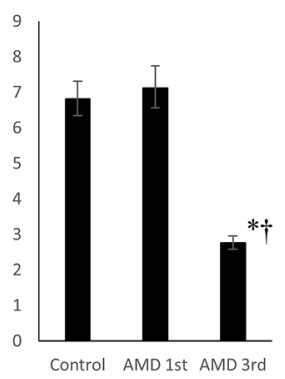

IP-10

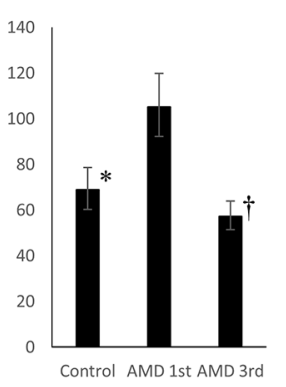

G

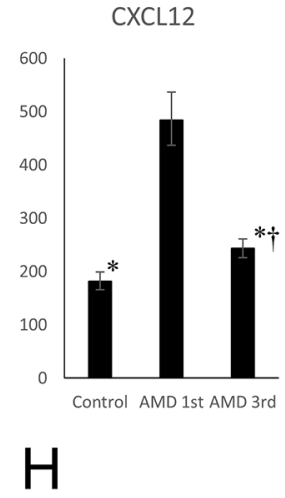

IL-10

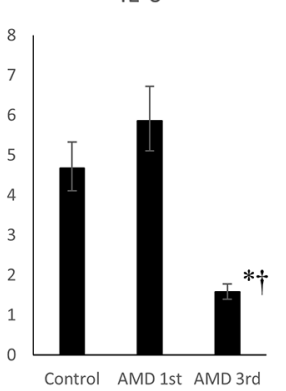

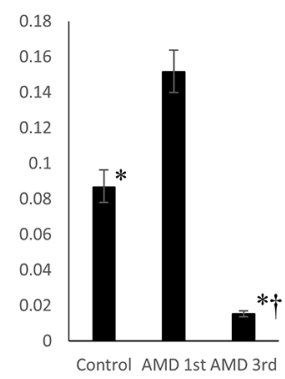

CXCL13

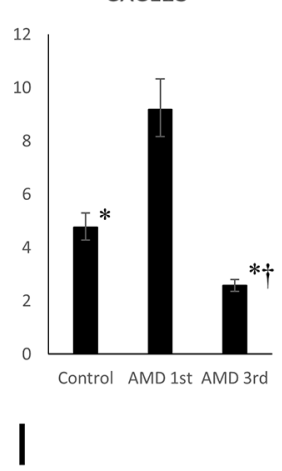

MMP-9
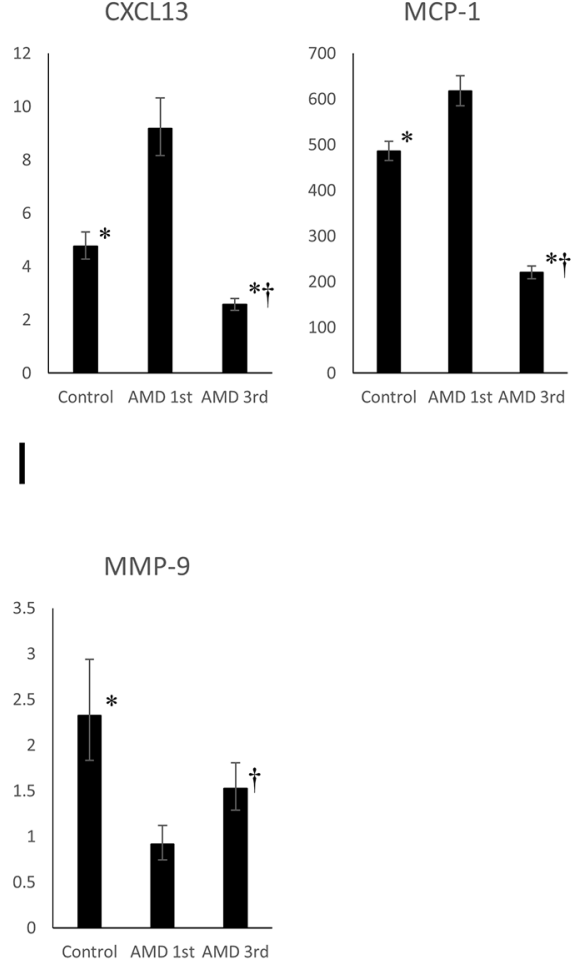

Figure 1 Cytokine concentrations in the aqueous humour of control and patients with AMD before the first ranibizumab injection and before the third ranibizumab injection. The concentrations of all cytokines except MMP-9 were significantly decreased after the two consecutive ranibizumab injections. Only MMP-9 was significantly increased. The third injection concentrations of CXCL1 $(p<0.0001), C X C L 13$ ( $p<0.0001)$, MCP-1 ( $<<0.0001)$, CCL11 ( $p<0.0001)$, IL-6 ( $p<0.0001)$ and IL-10 ( $p<0.0001)$ were significantly lower than those of the control. $(A)$ CXCL1: 10, 8.4 ( $p=0.25$ vs control), and 3.3 ( $p<0.0001$ vs control, $p<0.0001$ vs AMD 1st) (pg/mL). (B) IP-10: 69, 110 ( $p=0.017$ vs control), and 55 ( $p=0.48$ vs control, $p<0.0001$ vs AMD 1 st) (pg/mL). (C) CXCL12: 180, 480 ( $p<0.0001$ vs control), and 240 ( $p=0.044$ vs control, $p<0.0001$ vs AMD 1st) (pg/mL). (D) CXCL13: $4.8,9.2$ ( $p=0.0006$ vs control), and 2.6 ( $p<0.0001$ vs control, $p<0.0001$ vs AMD 1st) (pg/mL). (E) MCP-1: 490, 620 ( $p=0.0005$ vs control), and 220 ( $p<0.0001$ vs control, $p<0.0001$ vs AMD 1st) (pg/mL). (F) CCL11: 6.8, 7.1 ( $p=0.60$ vs control), and 2.8 ( $p<0.0001$ vs control, $p<0.0001$ vs AMD 1st) (pg/mL). (G) IL-6: $4.7,5.9$ $(p=0.27$ vs control), and $1.6(p<0.0001$ vs control, $p<0.0001$ vs $A M D 1 s t)(p g / m L)$. (H) IL-10: $0.087,0.15$ ( $p<0.0001$ vs control), and 0.015 ( $p<0.0001$ vs control, $p<0.0001$ vs AMD 1st) (pg/mL). (I) MMP-9: 2.3, 0.92 ( $p=0.0044$ vs control), and 1.5 ( $p=0.11$ vs control, $p=0.022$ vs AMD 1st) (pg/mL). * $p<0.05$ compared with 'control'. $+p<0.05$ compared with 'AMD 1st'. AMD, age-related macular degeneration; CCL11, C-C motif chemokine ligand 11; CXCL1, C-X-C motif chemokine ligand 1; CXCL12, C-X-C motif chemokine ligand 12; CXCL13, C-X-C motif chemokine ligand 13; IL-6, interleukin-6; IL-10; interleukin-10; IP-10, interferon- $\gamma$-induced protein 10; MCP-1, monocyte chemoattractant protein 1; MMP-9, matrix metalloproteinase 9.

stepwise variable selection (minimum Bayesian information criterion, increasing number of variables) was performed using age, sex, axial length, greatest linear dimension, PVD or not, disease duration, baseline CRT, and recurrence within 4 months of the first injection or not.

\section{RESULTS}

\section{Demographic characteristics of the patients}

The mean ages of the neovascular AMD and control cases were 73.0 (range, 54-89) and $74.8(55-87)$ years, respectively $(\mathrm{p}=0.17)$; 31 of the 48 neovascular AMD cases (64.6\%) and 37 of the 80 control cases $(46.3 \%)$ were men $(\mathrm{p}=0.043)$. The mean axial lengths of the neovascular AMD and control eyes were 23.8 and $23.2 \mathrm{~mm}$ $(\mathrm{p}=0.041)$, respectively. PVD was found in 22 patients with AMD $(45.3 \%)$ and 51 patients with cataract $(63.8 \%)(\mathrm{p}=0.048)$. No patients in the AMD group had newly developed PVD after two injections of ranibizumab. PCV was found in 27 of the 48 eyes. There was no retinal angiomatous proliferation (table 1$)$. Seven eyes were still wet at the third injection (tAMD, 5; PCV, 2). In addition,
25 eyes experienced recurrence within 4 months; the others showed recurrence over 6 months later or no recurrence during the 12-month observation period.

Cytokine concentrations in the aqueous humour of patients with AMD before the first and third ranibizumab injections The cytokine concentrations in the aqueous humour of the control and patients with AMD before treatment and before the third ranibizumab injection are shown in figure 1 . When compared with the samples taken before treatment, the concentrations of all cytokines except MMP-9 were significantly decreased after the two consecutive ranibizumab injections. Only MMP-9 was significantly increased. When compared with controls, the concentrations of CXCL1 $(p<0.0001)$, CXCL12 $(\mathrm{p}=0.044)$, CXCL13 $(\mathrm{p}<0.0001)$, MCP-1 $(\mathrm{p}<0.0001)$, CCL11 $(\mathrm{p}<0.0001)$, IL-6 $(\mathrm{p}<0.0001)$ and IL-10 $(\mathrm{p}<0.0001)$ were even lower 1 month after the second injection. Only the concentrations of IP-10 $(p=0.48)$ and MMP-9 $(p=0.11)$ were not significantly different from the controls. The cytokine concentrations before 
Table 2 Correlation between cytokine concentrations before treatment and before the third ranibizumab injection

\begin{tabular}{lll}
\hline & $\mathbf{R}$ & $\mathbf{p}$ Value \\
\hline CXCL1 & 0.51 & 0.0008 \\
IP-10 & 0.64 & $<0.0001$ \\
CXCL12 & 0.36 & 0.0131 \\
CXCL13 & 0.49 & 0.0004 \\
MCP-1 & 0.77 & $<0.0001$ \\
CCL11 & 0.53 & 0.0001 \\
IL-6 & 0.54 & $<0.0001$ \\
IL-10 & -0.11 & 0.4759 \\
MMP-9 & 0.35 & 0.0155 \\
\hline
\end{tabular}

$\mathrm{R}$, correlation coefficient.

CCL11, C-C motif chemokine ligand 11; CXCL1, C-X-C motif chemokine ligand 1;

CXCL12, C-X-C motif chemokine ligand 12; CXCL13, C-X-C motif chemokine ligand

13; IL-6, interleukin-6; IL-10; interleukin-10; IP-10, interferon- $\gamma$-induced protein 10;

MCP-1, monocyte chemoattractant protein 1; MMP-9, matrix metalloproteinase 9 .

treatment were correlated with those before the third ranibizumab injection, except for IL-10 (table 2). Regarding VEGF, we were not able to measure the concentrations in all the patients because the sample amount was not enough to measure the concentrations of both cytokines and VEGF. Thus, we measured VEGF concentrations in only $29(60 \%)$ cases before anti-VEGF therapy. The concentrations of VEGF were not correlated with those of other cytokines (online supplementary table 1).

\section{tAMD versus $P C V$}

There were no significant differences in age, sex, axial length, prevalence of PVD and cytokine concentrations between tAMD and PCV. The following changes in cytokine concentrations were seen in tAMD and PCV: CXCL12, $-45 \%$ and $-53 \%(\mathrm{p}=0.42)$; CXCL13, $-60 \%$ and $-79 \%(p=0.0034) ;$ IL-10, $-88 \%$ and $-91 \%(\mathrm{p}=0.34) ;$ IP-10, $-40 \%$ and $-50 \%(\mathrm{p}=0.40) ;$ IL-6, $-73 \%$ and $-73 \%(\mathrm{p}=0.98) ; \mathrm{MCP}-1,-62 \%$ and $-66 \% \quad(\mathrm{p}=0.29)$; CCL11, $-59 \%$ and $-63 \%(\mathrm{p}=0.59)$; CXCL1, $-68 \%$ and $-49 \%$ $(\mathrm{p}=0.054)$; and MMP-9, $+97 \%$ and $+47 \%(\mathrm{p}=0.50)$, respectively (figure 2). Note that only CXCL13 was significantly decreased in PCV compared with tAMD.

\section{Type 1 versus type 2 CNV}

There were no significant differences in age, sex, axial length, prevalence of PVD and cytokine concentrations between type 1 and type $2 \mathrm{CNV}$. The following changes in cytokine concentrations were seen in type 1 and type 2 CNV: CXCL12, $-35 \%$ and $-67 \%(\mathrm{p}=0.070) ;$ CXCL13, $-51 \%$ and $-80 \%(\mathrm{p}=0.042)$; IL-10, $-91 \%$ and $-76 \%(\mathrm{p}=0.082)$; IP- $10,-36 \%$ and $-50 \%$ $(\mathrm{p}=0.35)$; IL-6, $-74 \%$ and $-69 \%(\mathrm{p}=0.68) ; \mathrm{MCP}-1,-62 \%$ and $-63 \%(\mathrm{p}=0.82) ;$ CCL11, $-56 \%$ and $-69 \%(\mathrm{p}=0.24) ;$ CXCL1,
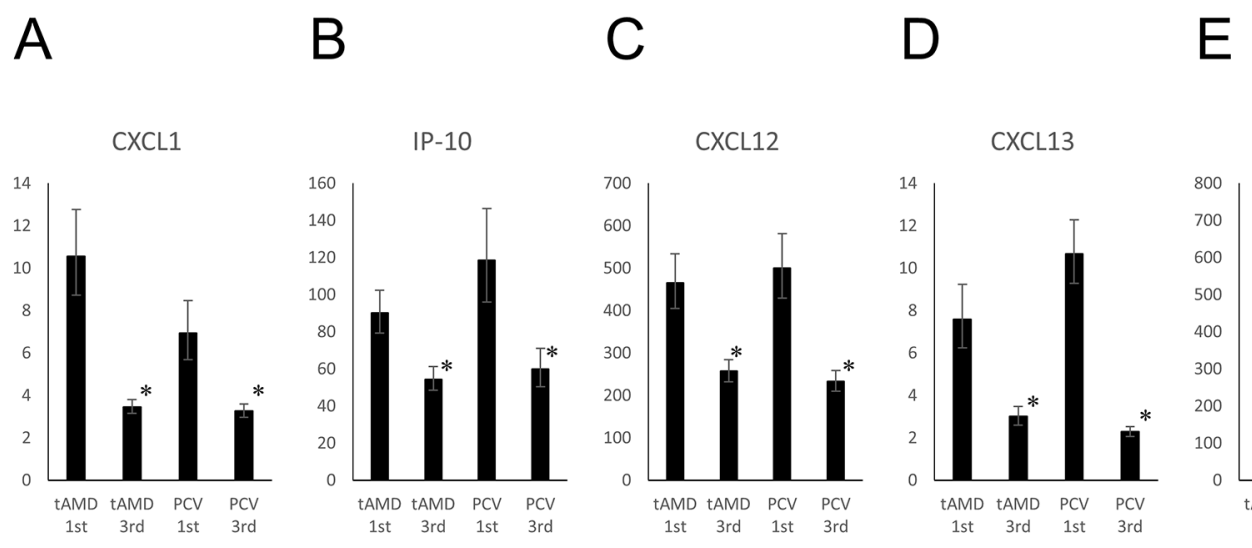

$\mathrm{F}$

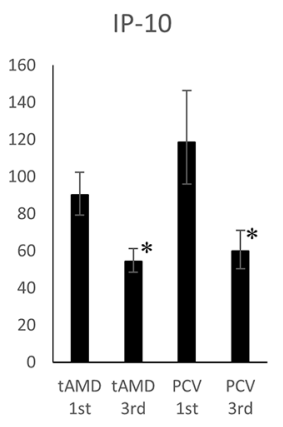

G

IL-6
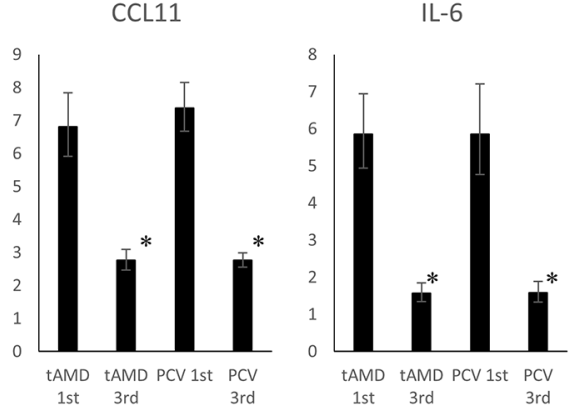

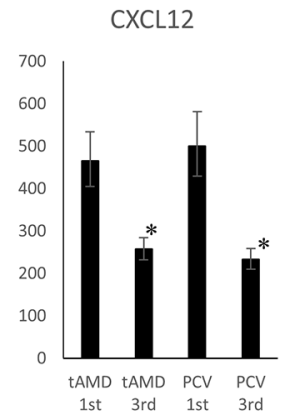

$\mathrm{H}$
CXCL13

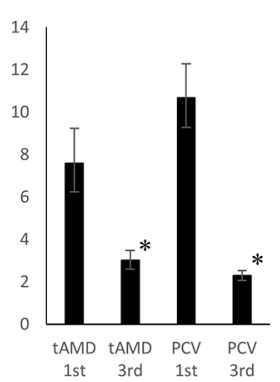

I

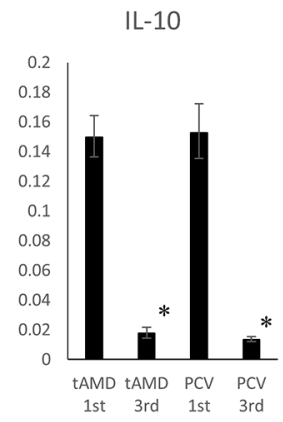

MMP-9

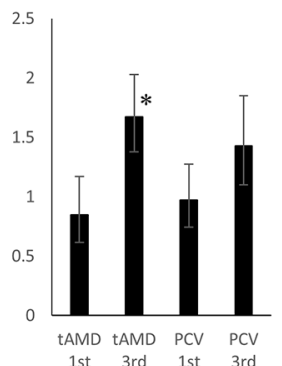

Figure 2 Reduction in cytokine concentrations in tAMD and PCV. Only CXCL13 was significantly decreased in PCV compared with tAMD. * $\mathrm{p}<0.05$ compared with 'tAMD/PCV first'. (A) CXCL1: $-68 \%(p<0.0001)$ and $-49 \%(p=0.0001)(p=0.054)$. (B) IP-10: $-40 \%(p<0.0001)$ and $-50 \%(p=0.0003)$ $(p=0.40)$. (C) CXCL12: $-45 \%(p=0.0013)$ and $-53 \%(p<0.0001)(p=0.42)$. (D) CXCL13: $-60 \%(p<0.0001)$ and $-79 \%(p<0.0001)(p=0.0034) .(E)$ MCP-1: $-62 \%(p<0.0001)$ and $-66 \%(p<0.0001)(p=0.29)$. (F) CCL11: $-59 \%(p<0.0001)$ and $-63 \%(p<0.0001)(p=0.59)$. (G) IL-6: $-73 \%(p<0.0001)$ and $-73 \%(p<0.0001)(p=0.98)$. (H) IL-10:-88\% ( $<<0.0001)$ and $-91 \%(p<0.0001)(p=0.34)$. (I) MMP-9: $+97 \%(p=0.046)$ and $+47 \%(p=0.21)$ $(p=0.50)$. CCL11, C-C motif chemokine ligand 11; CXCL, C-X-C motif chemokine ligand; IL, interleukin; IP-10, interferon- $\gamma$-induced protein 10; MCP-1, monocyte chemoattractant protein 1; MMP-9, matrix metalloproteinase 9; PCV, polypoidal choroidal vasculopathy; tAMD, third injection. 
Table 3 Comparison between eyes that achieved dry macula and those that did not at the third injection

\begin{tabular}{llll}
\hline & Dry & Wet & p Value \\
\hline CXCL1 & $8.8(6.4$ to 12$)$ & $6.6(3.3$ to 13$)$ & 0.45 \\
IP-10 & $110(82$ to 150$)$ & $85(42$ to 170$)$ & 0.50 \\
CXCL12 & $490(390$ to 620$)$ & $430(250$ to 740$)$ & 0.64 \\
CXCL13 & $8.8(6.8$ to 11$)$ & $12(6.3$ to 22$)$ & 0.38 \\
MCP-1 & $620(550$ to 690$)$ & $620(470$ to 820$)$ & 0.97 \\
CCL11 & $7.1(6.0$ to 8.6$)$ & $7.0(4.5$ to 11$)$ & 0.93 \\
IL-6 & $5.7(4.2$ to 7.7$)$ & $6.7(3.3$ to 14$)$ & 0.68 \\
IL-10 & $0.15(0.12$ to 0.18$)$ & $0.18(0.12$ to 0.27$)$ & 0.44 \\
\hline MMP-9 & $0.83(0.53$ to 1.3$)$ & $1.6(0.53$ to 4.6$)$ & 0.28 \\
\hline
\end{tabular}

Cytokine levels $(\mathrm{pg} / \mathrm{mL})$ are geometric mean $(95 \% \mathrm{Cl})$.

CCL11, C-C motif chemokine ligand 11; CXCL1, C-X-C motif chemokine ligand 1; CXCL12, C-X-C motif chemokine ligand 12; CXCL13, C-X-C motif chemokine ligand 13; IL-6, interleukin-6; IL-10; interleukin-10; IP-10, interferon- $\gamma$-induced protein 10; MCP-1, monocyte chemoattractant protein 1; MMP-9, matrix metalloproteinase 9 .

$-69 \%$ and $-64 \%(\mathrm{p}=0.78)$; and MMP-9, $+44 \%$ and $+435 \%$ $(p=0.079)$, respectively. Note that only CXCL13 was significantly decreased in type $2 \mathrm{CNV}$ compared with type $1 \mathrm{CNV}$.

Dry macula versus wet macula at month 3

There were no significant differences in the concentrations of any of the cytokines between the eyes that achieved dry macula and those that did not (table 3). Only the CXCL12 concentration at the first injection was significantly lower in the eyes that showed recurrence within 4 months compared with the other eyes ( 400 vs $600 \mathrm{pg} / \mathrm{mL}, \mathrm{p}=0.0406$ ). Of the variables analysedage, sex, axial length, greatest linear dimension, PVD or not, disease duration, number of annual injections, and recurrence within 4 months or not-only recurrence within 4 months or not was selected by stepwise variable selection as an explanatory variable for CXCL12 concentration at the first injection.

When initial CRT was examined as a response variable, explanatory variables, such as IP-10 (estimated value $=100, \mathrm{p}=0.0034$ ), were selected and significant.

\section{DISCUSSION}

All cytokines and chemokines upregulated in AMD-namely, IP-10, CXCL12, CXCL13, IL-10 and MCP-1-were decreased after anti-VEGF therapy. Using multiplex cytokine assays, the concentrations of IP-10, CXCL12, CXCL13, MCP-1 and IL-10 were shown to be higher in the aqueous humour of patients with exudative AMD after adjusting for age, sex and axial length. In contrast, the concentration of MMP-9 was found to be lower. MMP-9 is an enzyme expressed in the CNV membrane. ${ }^{15}$ Furthermore, we found that the concentrations of CXCL1, CCL11 and IL-6 also decreased after anti-VEGF therapy, although these cytokines were not elevated in AMD. Interestingly, the concentration of MMP-9 was increased after anti-VEGF therapy.

The concentrations of various cytokines were altered by antiVEGF therapy. The cytokine concentrations before treatment were correlated with those before third ranibizumab injection, except for IL-10. Before anti-VEGF treatment, the concentration of VEGF was not correlated with that of other cytokines (online supplementary table 1 ). We did not examine the concentrations of VEGF after anti-VEGF therapy because we recently found that intraocular VEGF concentrations are difficult to measure after anti-VEGF treatment. ${ }^{11}$
Previous studies have indicated that some intraocular cytokine concentrations associated with inflammatory are decreased after anti-VEGF therapy in patients with AMD, but there are several differences among these studies. Recent clinical studies in oncology determined that multiple circulating factors are modulated by VEGF inhibitors. ${ }^{16}{ }^{17}$ However, in ophthalmology, few investigations have focused on intraocular cytokine concentrations, despite the widespread use of anti-VEGF therapy for neovascular AMD. Previous studies showed that the aqueous humour concentrations of IL-2 and tumour necrosis factor (TNF)- $\alpha$ were decreased in patients with recurrent AMD who had received bevacizumab intraocular injections compared with control patients with cataract, ${ }^{2}$ suggesting that IL-2 and TNF- $\alpha$ are associated with disease activity. However, another study demonstrated that the concentrations of IL-1 $\beta$, IL-6, IL-8, IL-10, IL-12p and TNF- $\alpha$ did not significantly differ between patients with AMD who received bevacizumab injections within 3 months and those who did not. ${ }^{18}$ These apparent discrepancies might be due to the heterogeneity of patients with AMD. It has also been shown that the concentration of IL- 6 was stable after two bevacizumab injections, ${ }^{19}$ whereas another study showed that the concentrations of IL- 6 and IL- 8 were increased 2 days after bevacizumab injection. ${ }^{8}$ Thus, there seems to be a transient increase in IL- 6 and IL- 8 and possibly other inflammatory cytokines immediately after intravitreal anti-VEGF drug injection that levels off after several months.

It remains unclear whether each cytokine and chemokine plays different roles. IL-1 $\beta$, IL- 2 and IL- 6 are proinflammatory cytokines mainly produced by $\mathrm{T}$ lymphocytes or through the activation of inflammasomes, and TNF- $\alpha$ is a cytokine broadly associated with biological defence mechanisms via the activation of inflammation. IL-8 is a leucocyte chemotactic factor produced by leucocytes. IL-12 is a T lymphocyte-stimulating factor largely produced by $\mathrm{T}$ lymphocytes. On the other hand, IL-10 is an anti-inflammatory cytokine mainly produced by helper $\mathrm{T}$ lymphocytes and alternatively activated monocytes.

The findings of the current study show that concentrations of cytokines related to angiogenesis were decreased by antiVEGF therapy in patients with AMD. The primary finding of this study was that the concentrations of the previously reported cytokine IP-10 were also upregulated in eyes with neovascular AMD in Japanese individuals. ${ }^{3}$ A secondary and unexpected finding was that the concentrations of CXCL1, IP-10, CXCL12, CXCL13, MCP-1, CCL11, IL-6 and IL-10 were significantly lower after two consecutive injections than those in the controls. IP-10 was reported to be increased in a laser-induced mouse model of CNV, ${ }^{12}$ and serum IP-10 and CCL11 levels are elevated in patients with AMD. ${ }^{20}$ CCR3, a receptor of CCL11, is reported to be expressed in $\mathrm{CNV}$ endothelial cells and $\mathrm{CNV}$ was suppressed by CCR3 blockade. ${ }^{21}$ CXCL13 is upregulated in a laser-induced mouse model of $\mathrm{CNV}^{12}$ and in patients with AMD ${ }^{22}$ and CXCL13 inhibits the effects of fibroblast growth factor 2 (FGF-2) on endothelial cells. ${ }^{23}$ The levels of MCP-1 are reported to be higher in patients with AMD than in controls 8 and are involved in macrophage infiltration in mice with laser-induced $\mathrm{CNV}^{24}$ These results support the proangiogenic roles of IP-10, CCL11, CXCL13 and MCP-1, and the current study highlights the importance of anti-VEGF drugs in controlling their expression levels.

There are several explanations for these findings. First, the reduction may be a result of the inhibition of chronic inflammation in addition to the inflammation occurring at the CNV lesion. Indeed, several intraocular cytokine levels are elevated in healthy older subjects compared with younger subjects. 
Histological studies have revealed that chronic inflammation is generally considered to occur at the retinal pigment epithelial/choroidal interface in eyes with early signs of AMD, such as drusen. ${ }^{25}$ Furthermore, in exudative AMD, there is a more prominent upregulation of inflammatory cytokines/chemokines from retinal pigment epithelium cells and macrophages/ monocytes, which positively and negatively control CNV activity. Second, recent clinical studies suggest that anti-VEGF therapy affects the choroid. Anti-VEGF therapy decreases choroidal thickness and there is an association between choroidal hyperpermeability and several cytokines. ${ }^{14}$ Interestingly, alternatively or classically activated macrophages/ monocytes express most of the cytokines and chemokines investigated in the current study. Although further mechanistic studies are needed, we assume that anti-VEGF drugs block VEGF signalling and VEGF-dependent chemotaxis as well, as has been shown by laboratory studies, ${ }^{12212324}$ thereby reducing the inflammatory reaction presumably induced by recruited monocytes/macrophages.

The therapeutic effect of the alterations in cytokine concentrations by anti-VEGF therapy was not clear in the short term. In this study, there were no significant differences in any cytokine concentrations between the eyes that remained wet at the third injection and those that did not. Although anti-VEGF therapy showed an adequate therapeutic effect on AMD, the suppression of VEGF may not be sufficient for the suppression of CNV activity in the short term. When the concentration of VEGF is maintained at low levels for longer periods, low concentrations of cytokines would also be maintained and CNV activity might be suppressed.

We investigated the effect of cytokines between different types of AMD. There were no significant differences in cytokine concentrations, except CXCL13, between tAMD and PCV. Additionally, there were significant differences in cytokine concentrations, such as IP-10, MCP-1, CXCL-12 and IL- 6 between type $1 \mathrm{CNV}$ and type $2 \mathrm{CNV}$ in tAMD. All cytokine concentrations were lower in type $1 \mathrm{CNV}$ than in type $2 \mathrm{CNV}$. Because type $1 \mathrm{CNV}$ existed under the retinal pigmentary epithelium, it is rational to consider that cytokines secreted from type $1 \mathrm{CNV}$ could not spread easily in vitreous and aqueous humour. Before anti-VEGF therapy, CRT was not correlated with cytokine concentrations, except for IP-10. The cytokines investigated in the current study did not influence CRT directly.

There are some limitations to this study. First, this is a singlecentre study and the patient selection may be biased. Second, all of the subjects included in this study were Japanese. Thus, further studies are needed to confirm our results in other ethnicities. Third, we were not able to measure the concentration of VEGF in all cases, which might have introduced bias in the correlation study. The multiplex platform we used appeared to overestimate the cytokine concentrations in most samples. However, the main purpose of the study was to compare concentrations between the first and post-treatment samples. Thus, the impact of overestimation should be limited.

\section{CONCLUSION}

The concentrations of the cytokines IP-10, CXCL12, CXCL13, IL-10 and MCP-1, which were elevated in AMD, decreased after anti-VEGF therapy. The concentrations of CXCL1, CCL11 and IL-6, which were not elevated in AMD, were also decreased after anti-VEGF therapy. The concentration of MMP-9, which was decreased in AMD, was increased after anti-VEGF therapy.
Twitter@takaha4

Contributors HT designed the study, collected and analysed data, and drafted the manuscript. YY designed the study and revised the manuscript. SS, XT, YI, YN, YA, YF and $\mathrm{HK}$ revised the manuscript.

Funding This work was supported by a KAKENHI grant from the Japan Society for the Promotion of Science, Grant Number 15K10899.

Competing interests Shinichi Sakamoto, Xue Tan, Yoko Nomura, Yusuke Arai: None. Hidenori Takahashi: Lecturer's fees from Kowa Pharmaceutical, Novartis Pharmaceuticals, Bayer Yakuhin, and Santen Pharmaceutical, educational presentation fee from Tochigi Prefectural Ophthalmologists Association, grants from Bayer Yakuhin and Novartis Pharma, unrelated to this work. Yujilnoue: Lecturer's fees from Kowa Pharmaceutical, Novartis Pharmaceuticals, Bayer Yakuhin, and Santen Pharmaceutical, educational presentation fee from Tochigi Prefectural Ophthalmologists Association, unrelated to this work. YujiroFujino: Lecturer's fees from ALCON JAPAN LTD. and Otsuka Pharmaceutical, educational presentation fee from Tokyo Association of Ophthalmologists, unrelated to this work. Hidetoshi Kawashima: Lecturer's fees from Kowa Pharmaceutical, Novartis Pharmaceuticals, and Santen Pharmaceutical, educational presentation fee from Tochigi Prefectural Ophthalmologists Association, unrelated to this work. Yasuo Yanagi: Lecturer's fees from Novartis Pharmaceuticals, Bayer Yakuhin, MSD, and Santen Pharmaceutical, grants from Bayer Yakuhin, Santen Pharmaceutical, and Novartis Pharma, advisory board member for Novartis Pharmaceuticals and Bayer Yakuhin, unrelated to this work.

Patient consent Obtained.

Ethics approval $\mathrm{JCHO}$.

Provenance and peer review Not commissioned; externally peer reviewed.

Open Access This is an Open Access article distributed in accordance with the Creative Commons Attribution Non Commercial (CC BY-NC 4.0) license, which permits others to distribute, remix, adapt, build upon this work non-commercially, and license their derivative works on different terms, provided the original work is properly cited and the use is non-commercial. See: http://creativecommons.org/ licenses/by-nc/4.0/

(c) Article author(s) (or their employer(s) unless otherwise stated in the text of the article) 2018. All rights reserved. No commercial use is permitted unless otherwise expressly granted.

\section{REFERENCES}

1 Funk M, Karl D, Georgopoulos M, et al. Neovascular age-related macular degeneration: intraocular cytokines and growth factors and the influence of therapy with ranibizumab. Ophthalmology 2009;116:2393-9.

2 Roh MI, Kim HS, Song JH, et al. Concentration of cytokines in the aqueous humor of patients with naive, recurrent and regressed CNV associated with amd after bevacizumab treatment. Retina 2009;29:523-9.

3 Sakurada Y, Nakamura Y, Yoneyama S, et al. Aqueous humor cytokine levels in patients with polypoidal choroidal vasculopathy and neovascular age-related macular degeneration. Ophthalmic Res 2015;53:2-7.

4 Strieter RM, Wiggins R, Phan SH, et al. Monocyte chemotactic protein gene expression by cytokine-treated human fibroblasts and endothelial cells. Biochem Biophys Res Commun 1989;162:694-700.

5 Nagasawa T, Hirota S, Tachibana K, et al. Defects of B-cell lymphopoiesis and bone-marrow myelopoiesis in mice lacking the CXC chemokine PBSF/SDF-1. Nature 1996:382:635-8.

6 Steen B, Sejersen S, Berglin L, et al. Matrix metalloproteinases and metalloproteinase inhibitors in choroidal neovascular membranes. Invest Ophthalmo/ Vis Sci 1998:39:2194-200.

7 Rezar-Dreindl S, Sacu S, Eibenberger K, et al. The intraocular cytokine Profile and therapeutic response in persistent neovascular Age-Related Macular Degeneration. Invest Ophthalmol Vis Sci 2016;57:4144-50.

8 Agawa T, Usui Y, Wakabayashi Y, et al. Profile of intraocular immune mediators in patients with age-related macular degeneration and the effect of intravitreal bevacizumab injection. Retina 2014;34:1811-8.

9 Ziemssen F, Eter N, Fauser S, et al. [Retrospective investigation of anti-VEGF treatment reality and effectiveness in patients with neovascular age-related macular degeneration (AMD) in Germany: treatment reality of ranibizumab for neovascular AMD in Germany]. Ophthalmologe 2015;112:246-54.

10 Salnikov AV, Heldin NE, Stuhr LB, et al. Inhibition of carcinoma cell-derived VEGF reduces inflammatory characteristics in xenograft carcinoma. Int J Cancer 2006;119:2795-802.

11 Takahashi H, Nomura Y, Nishida J, et al. Vascular endothelial growth factor (VEGF) Concentration is underestimated by Enzyme-Linked Immunosorbent assay in the presence of Anti-VEGF drugs. Invest Ophthalmo/ Vis Sci 2016;57:462-6.

12 Fujimura S, Takahashi H, Yuda K, et al. Angiostatic effect of CXCR3 expressed on choroidal neovascularization. Invest Ophthalmol Vis Sci 2012;53:1999-2006. 
13 Takahashi H, Nomura Y, Tan X, et al. Effects of posterior vitreous detachment on aqueous humour levels of VEGF and inflammatory cytokines. Br J Ophthalmol 2015;99:1065-9.

14 Nomura Y, Takahashi H, Fujino Y, et al. Association between aqueous humor cxc motif chemokine ligand 13 levels and subfoveal choroidal thickness in normal older subjects. Retina 2016;36:192-8.

15 Jonas JB, Tao Y, Neumaier M, et al. Cytokine concentration in aqueous humour of eyes with exudative age-related macular degeneration. Acta Ophthalmol 2012;90:e381-e388.

16 Ebos JM, Lee CR, Christensen JG, et al. Multiple circulating proangiogenic factors induced by sunitinib malate are tumor-independent and correlate with antitumor efficacy. Proc Natl Acad Sci U S A 2007;104:17069-74.

17 Hanrahan EO, Lin HY, Kim ES, et al. Distinct patterns of cytokine and angiogenic facto modulation and markers of benefit for vandetanib and/or chemotherapy in patients with non-small-cell lung Cancer. J Clin Oncol 2010;28:193-201.

18 Miao H, Tao Y, Li XX, XX L. Inflammatory cytokines in aqueous humor of patients with choroidal neovascularization. Mol Vis 2012;18:574-80.

19 Chalam KV, Grover S, Sambhav K, et al. Aqueous interleukin-6 levels are superior to vascular endothelial growth factor in predicting therapeutic response to Bevacizumab in age-related macular degeneration. J Ophthalmol 2014;2014:1-6.
20 Mo FM, Proia AD, Johnson WH, et al. Interferon gamma-inducible protein-10 (IP-10) and eotaxin as biomarkers in age-related macular degeneration. Invest Ophthalmol Vis Sci 2010;51:4226-36.

21 Takeda A, Baffi JZ, Kleinman ME, et al. CCR3 is a target for age-related macular degeneration diagnosis and therapy. Nature 2009;460:225-30.

22 Silveira AC, Morrison MA, Ji F, et al. Convergence of linkage, gene expression and association data demonstrates the influence of the RAR-related orphan receptor alpha (RORA) gene on Neovascular AMD: a systems biology based approach. Vision Res 2010;50:698-715.

23 Spinetti G, Camarda G, Bernardini G, et al. The chemokine CXCL13 (BCA-1) inhibits FGF-2 effects on endothelial cells. Biochem Biophys Res Commun 2001;289:19-24.

24 Yamada K, Sakurai E, Itaya M, et al. Inhibition of laser-induced choroidal neovascularization by atorvastatin by downregulation of monocyte chemotactic protein-1 synthesis in mice. Invest Ophthalmol Vis Sci 2007;48:1839-43.

25 Hageman GS, Luthert PJ, Victor Chong NH, et al. An integrated hypothesis that considers drusen as biomarkers of immune-mediated processes at the RPE-Bruch's membrane interface in aging and age-related macular degeneration. Prog Retin Eye Res 2001;20:705-32. 